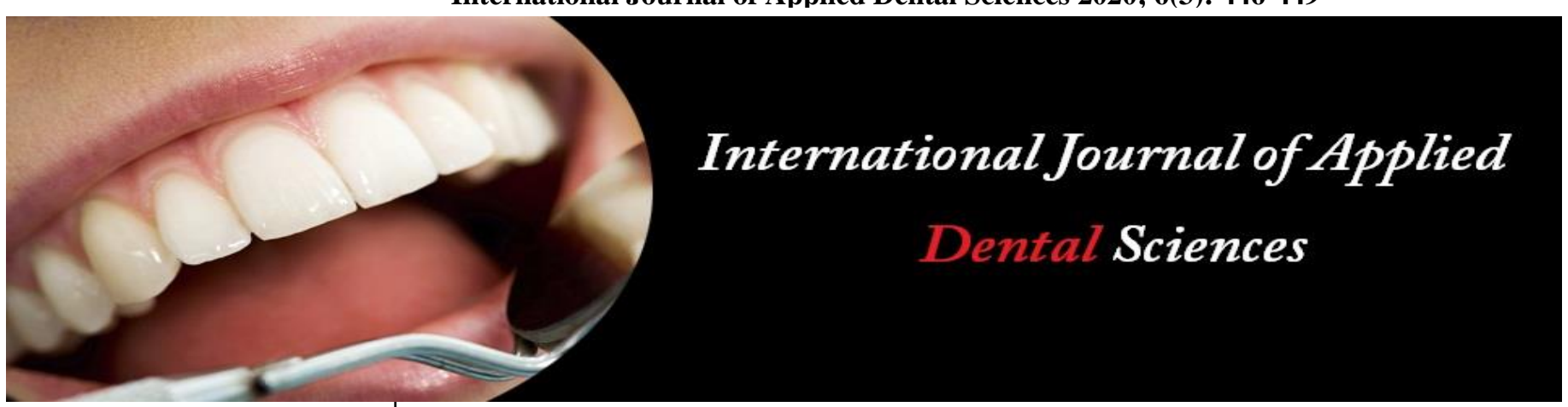

ISSN Print: 2394-7489

ISSN Online: 2394-7497

IJADS 2020; 6(3): 446-449

(C) 2020 IJADS

www.oraljournal.com

Received: 08-05-2020

Accepted: 10-06-2020

Dr. Sami Faisal Jamda BDS, MDS, (Maxillofacial surgery), MD (General Medicine), Specialist Maxillofacial surgeon, Ministry of health, Hafar Al Batin Central Hospital, Saudi Arabia

Dr. Asna Fatima Jamdar Post Graduate Resident, Department of orthodontics and Dentofacial Orthopaedics, Alameen dental college and hospital, Vijayapura, Karnataka, India
Corresponding Author: Dr. Sami Faisal Jamdar BDS, MDS, (Maxillofacial surgery), MD (General Medicine), Specialist Maxillofacial surgeon, Ministry of health, Hafar Al Batin Central Hospital, Saudi Arabia

\section{Lymph nodes assessment of neck in oral squamous cell carcinoma and its implication in management}

\section{Dr. Sami Faisal Jamdar and Dr. Asna Fatima Jamdar}

DOI: https://doi.org/10.22271/oral.2020.v6.i3g.989

\section{Abstract}

Background and Aim: This study was conducted to find the correlation between clinically palpable neck nodes and their histopathological examination results which gives some information to the clinician regarding the choice of treatment and for appropriate management of the neck. After the surgical procedure, the whole specimen was submitted for the histopathological examination and a correlation between clinical positive or negative nodes and their histologically confirmed malignancies were drawn. Materials \& Methods: A total of 200 patients with the diagnosis of oral malignancy subjected to oral and maxillofacial surgery department and institute of oncology over the period of 2 years were included in the study. Once the confirmation by histopathology was done, routine blood investigation, HIV/HBSAg test, ECG was done and physician's consent was taken prior to surgery under general anaesthesia. Pre-operatively thorough clinical palpation of the five levels of cervical lymph nodes was performed bilaterally.

Results: On clinical examination of lymph nodes by palpation in 200 cases, 50 sides of necks were negative for palpation and 162 sides of necks were positive for palpation. On the histopathological examination of neck dissections, 8 of necks were positive. That is 46 cases were true positive that the histopathology showed no metastasis and 8 cases were false positive which showed metastasis in histopathology.

Conclusion: Histopathology examination with its ability to detect $100 \%$ metastases in cervical lymph nodes remains the gold standard. Even though, Clinical examination was and is still the routine first line in detecting cervical lymph node metastases in head and neck squamous cell carcinoma, Its use shall be restricted for only an initial evaluation of the patient.

Keywords: Neck, lymph nodes, OSCC, histopathology

\section{Introduction}

Oral cancer includes all malignant neoplasm affecting the oral cavity, principally the oral mucosa. SCC is the most common neoplasm and comprises $90 \%$ to $95 \%$ of all oral malignancies. Cancers of mouth and pharynx account for 363000 new cases and 200000 deaths annually worldwide ${ }^{[1]}$. Most oral cancers are squamous cell carcinomas because the mutagens in tobacco, alcohol and viruses have prolonged exposure to the superficial layers of oral mucosa ${ }^{[2,3]}$.

Oral cancer is a disease of increasing age: approximately $95 \%$ of cases occur in people older than 45 years. The majority of the oral cancers involve the tongue, oropharynx and the floor of the mouth. The lips, gingiva, dorsal tongue and palate are less common sites. Metastasis is the process whereby primary tumor cells spread to distant sites in the body and is the leading cause of cancer-related deaths ${ }^{[4]}$.

Metastases are tumor implants discontinuous with the primary tumor. Metastasis unequivocally marks a tumor as malignant because benign, neoplasm do not metastasize. The invasiveness of cancers permits them to penetrate into blood vessels, lymphatics, and body cavities, providing the opportunity for spread. With few exceptions, all cancers can metastasize ${ }^{[5]}$.

Pre operative assessment of the metastatic status of cervical lymph nodes is a well-recognized problem in the management of patients with oral cancer. The difficulty in predicting the presence of metastatic disease in clinically negative neck has led to wide spread use of elective 
(prophylactic) treatment of neck, by either neck dissection or radiotherapy ${ }^{[6]}$. Currently, several methods are available for investigating the presence and extent of nodal metastasis like CT, MRI, PET etc however, there is still much doubt of efficiency of these investigations. False negative incidence rates as low as $0-14 \%$ have been reported and on the basis of such reports, the rationale of elective neck dissection has been questioned $^{[7]}$.

In addition pre-operative assessment of the cervical lymph node status helps in planning suitable surgical management of the neck, wherein the justification to operate the neck is being questioned more often than not, owing to the fact that only about $30 \%$ of clinically negative necks are histopathologically positive once operated ${ }^{[8]}$.

This suggests that $70 \%$ of patients are treated unnecessarily to benefit the other $30 \%$. The pre operative assessment and even the advent of highly reliable imaging techniques could alter this situation, such that the number of elective treatments would be greatly reduced or eliminated ${ }^{[9]}$.

This study was conducted to find the correlation between clinically palpable neck nodes and their histopathologic examination results which gives some information to the clinician regarding the choice of treatment and for appropriate management of the neck. After the surgical procedure, the whole specimen was submitted for the histopathological examination and a correlation between clinical positive or negative nodes and their histologically confirmed malignancies were drawn and submitted for statistical analysis.

\section{Materials \& Methods}

A total of 200 patients with the diagnosis of oral malignancy subjected to oral and maxillofacial surgery department and institute of oncology over the period of 2 years were included in the study. The ethical clearance certificate was obtained from the institute ethical committee. All the patients were informed about the study and the consent letter was signed by each one of them.

All the patients included for the study were previously not treated for oral squamous cell carcinoma; treatment planned was surgical ablation for oral squamous cell carcinoma. Male and female both were included in the study. Patients who were previously treated for oral squamous cell carcinoma, who had received radiotherapy, presence of distant metastasis at times of presentation and those who are unfit for surgery were excluded from the study.

A detailed history was recorded. A thorough clinical examination, including systemic examination was done and the clinical sign and symptoms were recorded. For the confirmation of oral squamous cell carcinoma an incisional biopsy was done. Based on the clinical requirement the CT, MRI was taken.

Once the confirmation by histopathology was done, routine blood investigation, HIV/HBSAg test, ECG was done and physician's consent was taken prior to surgery under general anaesthesia. Pre-operatively thorough clinical palpation of the five levels of cervical lymph nodes was performed bilaterally. The site, size, consistency and fixity of all the clinically palpable nodes were documented according to AJCC/UICC staging criteria.

A total of 200 neck dissections were done in total of 200 patients; among which radical neck dissection was performed in 64 patients, supraomohyoid neck dissection was performed in 82 patients, functional neck dissection was performed in 8 patients, modified radical neck dissection type-I was performed in 2 patient, modified radical neck dissection typeII was performed in 12 patients. Modified radical neck dissection type-III was performed in 20 patients. The resected neck specimens were oriented anatomically and markings for each lymph node level were done and sent for the histopathological examination. Descriptive statistical analysis has been carried out in the present study. Chi-square test has been used to find the significance of data to analyze cervical lymph node metastasis.

\section{Results}

The present study included 200 patients of oral SCC. Al the included patients were diagnosed histopathological with oral squamous cell carcinoma. The present study was done with the aim to correlate the clinical palpation of lymph node with histopathological findings. The age of the patients included in the study ranges from 25 to 80 years with mean age of 52 years. The gender distribution in the study stated there were 132 males and 68 females.

In the analysis of primarily site involvement, there was more predilections for buccal mucosa, 84 patients had site of buccal mucosa, 48 cases showed involvement of tongue, 32 cases were of GB sulcus involvement, 10 cases involved retro molar trigone, 10 cases were of floor of mouth, 10 cases involving hard palate and 2 cases of lower lip. (Table 1)

Table 1: Site of the tumor

\begin{tabular}{|c|c|}
\hline Site of tumor & Number $(\mathbf{n}=\mathbf{2 0 0})$ \\
\hline Buccal mucosa & 84 \\
\hline Tongue & 48 \\
\hline GB sulcus & 32 \\
\hline Floor of mouth & 10 \\
\hline Retro molar trigone & 10 \\
\hline Floor of mouth & 10 \\
\hline Hard palate & 10 \\
\hline Lower lip & 2 \\
\hline
\end{tabular}

On clinical examination of lymph nodes by palpation in 200 cases, 50 sides of necks were negative for palpation and 162 sides of necks were positive for palpation. On the histopathological examination of neck dissections, 8 of necks were positive. That is 46 cases were true positive that the histopathology showed no metastasis and 8 cases were false positive which showed metastasis in histopathology.

According to the Levels of Lymph nodes wise, 154 Level I lymph nodes, 72 Level II lymph nodes, 8 Level III lymph nodes and 2 Level IV lymph nodes were positive for metastasis on palpation. The histopathological examination of the neck showed metastasis in 86 Levels I lymph nodes, 48 Level II lymph nodes, 14 Level III lymph nodes, 4 Level IV lymph nodes. Both on clinical palpation and on histologically, Level V lymph nodes were not palpable in all our cases.

Table 2: Level of lymph nodes

\begin{tabular}{|c|c|c|}
\hline Levels of lymph nodes & Clinical & Histopathological \\
\hline Level I & 154 & 84 \\
\hline Level II & 76 & 48 \\
\hline Level III & 8 & 14 \\
\hline Level IV & 2 & 4 \\
\hline Level V & 0 & 0 \\
\hline
\end{tabular}


Table 3: Clinical palpation with histopathological examination

\begin{tabular}{|c|c|c|c|c|c|}
\hline Clinical V/S Histopathological & True positive & False positive & False negative & True negative & Total \\
\hline Level I & 84 & 70 & 2 & 44 & 200 \\
\hline Level II & 36 & 36 & 12 & 116 & 200 \\
\hline Level III & 6 & 2 & 8 & 184 & 200 \\
\hline Level IV & 0 & 2 & 4 & 194 & 200 \\
\hline Level V & - & - & - & - & - \\
\hline
\end{tabular}

In level I nodes there is sensitivity of $97.7 \%$ and specificity $44.4 \%$ and the overall accuracy of clinical palpation analyses was of $66 \%$. In level II nodes there is sensitivity of $75 \%$ and specificity $78 \%$ and the overall accuracy of clinical palpation analyses of $77.4 \%$. In level III nodes there is sensitivity of $42.9 \%$ and specificity $98.9 \%$ and the overall accuracy of clinical palpation analyses of $95.3 \%$. In level IV nodes there is sensitivity of $0 \%$ and specificity $99 \%$ and the overall accuracy of clinical palpation analyses of $97.2 \%$. The overall Accuracy of clinical palpation with histopathology in all levels of lymph nodes (Level I to V) is $67 \%$.

\section{Discussion}

Squamous cell carcinoma is the most commonly encountered malignant neoplasm in the upper aero digestive tract, accounting for more than $90 \%$ of reported oral cancer. It is the sixth most commonly reported cancer worldwide. Men are affected twice as often as women and most patients are older than 45 years of age ${ }^{[10]}$.

The presence of cervical metastatic nodal disease is a major prognostic determinant for patients with oral cavity cancer, significantly reducing patient survival. Metastases are tumor implants discontinuous with the primary tumor. Metastasis unequivocally marks a tumor as malignant because benign neoplasms do not metastasize ${ }^{[11]}$. The invasiveness of cancers permits them to penetrate into blood vessels, lymphatics, and body cavities, providing the opportunity for spread. With few exceptions, all cancers can metastasize. The major exceptions are most malignant neoplasms of the glial cells in the central nervous system, called gliomas, and basal cell carcinomas of the skin, rarely metastasize ${ }^{[12]}$.

Clinical palpation is the routine first line in evaluating metastatic cervical lymphadenopathy, giving information regarding the size, location and number of palpable lymph nodes. In addition to providing data for the $\mathrm{N}$-status as part of overall TNM staging, clinical examination plays a role in planning treatment for the neck. Of greatest concern is whether palpation is accurate in diagnosing metastatic lymph nodes ${ }^{[13]}$

Several authors have reported an incidence of false negative findings as low as $0-14 \%$ have been reported. Atula et al. ${ }^{[14]}$ have reported a $15-25 \%$ risk of occult metastasis, in spite of a normal palpation finding.

Fischbein et al. ${ }^{[15]}$ have found clinical examination to have only $70 \%$ accuracy at best. The positive predictive values as reported in literature varies from $78-92 \%$.

In our series of 200 neck dissections in 200 cases, the findings of study of correlating of clinical palpitation of lymph nodes levels with histopathology are.

Over all incidence of cervical metastasis of 102 specimens were histologically positive and similar of kind results were shown by $\mathrm{Li}$ et al. with overall incidence of $45 \%$ cervical metastasis in 153 cases.

Out of total 162 cases which were positive on clinical palpation, 98 Specimens showed positive on histopathology. Out of total 50 N0 necks, Histopathology showed 8 specimens were positive.
The overall accuracy of clinical palpation with histopathology in all levels of lymph nodes (Level I to V) is $63 \%$ which is similar result showed by Fischbein et al. ${ }^{[15]}$ On Clinical palpation, sensitivity decreases gradually from Level I to Level III and Level IV and Level V sensitivity was 0. Our series showed the \% of metastasis in Level I is $37 \%$, Level II is $27.8 \%$, Level III is $7.4 \%$ and in Level IV is $3.5 \%$ and Level $\mathrm{V}$ showed no metastasis an these results are correlating with other studies by Shah ${ }^{[16]}$, Lindberg ${ }^{[17]}$, Woolgar ${ }^{[18]}$.

\section{Conclusion}

Histopathology examination with its ability to detect $100 \%$ metastases in cervical lymph nodes remains the gold standard. Even though, Clinical examination was and is still the routine first line in detecting cervical lymph node metastases in head and neck squamous cell carcinoma, Its use shall be restricted for only an initial evaluation of the patient.

\section{References}

1. Sudhakara Reddy K. Clinico-histopathological correlation of neck nodes in oral squamous cell carcinoma and its implication in management, 2009.

2. Reis SRdA, Santo E, Andrade MGS, Sadigursky M. Cytologic alterations in the oral mucosa after chronic exposure to ethanol. Brazilian oral research. 2006; 20:97102.

3. Metgud R, Astekar M, Verma M, Sharma A. Role of viruses in oral squamous cell carcinoma. Oncology reviews, 2012, 6 .

4. Llewellyn C, Johnson N, Warnakulasuriya K. Risk factors for squamous cell carcinoma of the oral cavity in young people - a comprehensive literature review. Oral oncology. 2001; 37:401-18.

5. Nicolson GL, Poste G. Tumor implantation and invasion at metastatic sites. Int Rev Exp Pathol. 1983; 25:77-181.

6. Wide J, White D, Woolgar J, Brown J, Vaughan E, Lewis-Jones $H$. Magnetic resonance imaging in the assessment of cervical nodal metastasis in oral squamous cell carcinoma. Clinical radiology. 1999; 54:90-4.

7. Lee J. Electronic Poster Viewing-80 selected posters EP1.

8. Sadat SA, Rita RN, Zaman A-U, Rob MA, Husain MM, Ahmed M. High Resolution Sonography in Diagnosis of Metastatic Cervical Lymph Nodes in Oral Squamous Cell Carcinoma. Journal of Bangladesh College of Physicians and Surgeons. 2010; 28:92-9.

9. Patel T. Surgery in the patient with liver disease. Mayo Clinic Proceedings: Elsevier, 1999, 593-9.

10. Walker DM, Boey G, McDonald L. The pathology of oral cancer. Pathology. 2003; 35:376-83.

11. Barresi V, Bonetti LR, Bettelli S, Maiorana A. XXXI International Congress of the IAP and 28th Congress of the ESP. Virchows Arch. 2016; 469:S1-S346.

12. Sherbet R. Metastatic Spread of Cancer: Springer, 2015.

13. Cole DJ, Baron PL, O'brien PH. Multi-marker RT-PCR panel for detecting metastatic breast cancer. Google Patents, 2000. 
14. Atula TS, Varpula MJ, Kurki TJ, Klemi P-J, Grénman R. Assessment of cervical lymph node status in head and neck cancer patients: palpation, computed tomography and low field magnetic resonance imaging compared with ultrasound-guided fine-needle aspiration cytology. European journal of radiology. 1997; 25:152-61.

15. Fischbein NJ, Noworolski SM, Henry RG, Kaplan MJ, Dillon WP, Nelson SJ. Assessment of metastatic cervical adenopathy using dynamic contrast-enhanced MR imaging. American Journal of Neuroradiology. 2003; 24:301-11.

16. Shah JP, Andersen PE. The impact of patterns of nodal metastasis on modifications of neck dissection. Annals of surgical oncology. 1994; 1:521-32.

17. Lindberg $R$. Distribution of cervical lymph node metastases from squamous cell carcinoma of the upper respiratory and digestive tracts. Cancer. 1972; 29:1446-9.

18. Woolgar JA, Vaughan E, Scott J, Brown J. Pathological findings in clinically false-negative and false-positive neck dissections for oral carcinoma. Annals of the Royal College of Surgeons of England. 1994; 76:237. 